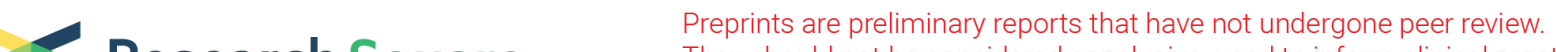 $\begin{array}{ll}\text { Research Square } & \text { They should not be considered conclusive, used to inform clinical practice, } \\ \text { or referenced by the media as validated information. }\end{array}$
}

\section{A low cartilage formation and repair endotype predicts radiographic progression of symptomatic knee osteoarthritis}

\author{
Jonathan Samuels \\ New York University \\ Svetlana Krasnokutsky \\ New York University \\ Inger Byrjalsen \\ Nordic Bioscience \\ Virginia B. Kraus \\ Duke University \\ Yi He \\ Nordic Bioscience \\ Morten A. Karsdal \\ Nordic Bioscience \\ Steven B. Abramson \\ New York University \\ Mukundan Attur \\ New York University \\ Anne C. Bay-Jensen \\ Nordic Bioscience
}

Yunyun Luo ( $\nabla$ yyl@nordicbio.com )

Nordic Bioscience https://orcid.org/0000-0002-4515-5888

\section{Research article}

Keywords: cartilage biomarker, extracellular matrix, joint space narrowing, knee osteoarthritis, matrix synthesis

Posted Date: April 9th, 2020

DOI: https://doi.org/10.21203/rs.3.rs-21457/v1

License: (c) (1) This work is licensed under a Creative Commons Attribution 4.0 International License. Read Full License 
Version of Record: A version of this preprint was published at Journal of Orthopaedics and Traumatology on March 9th, 2021. See the published version at https://doi.org/10.1186/s10195-021-00572-0. 


\section{Abstract}

Objective: Osteoarthritis (OA) is a disease with multiple endotypes. A hallmark of OA is loss of cartilage; however, it is evident that the rate of cartilage loss differs among patients, which may partly be attributed to differential capacity for cartilage repair. We hypothesize that a low cartilage repair endotype exists and that such endotypes are more likely to progress radiographically. The aim of this study was to examine the associations of level of cartilage formation with OA severity and radiographic OA progression. We used the blood-based marker PRO-C2, reflecting type II collagen formation, to assess levels of cartilage formation.

Methods: The type II collagen propeptide PRO-C2 was measured in the serum/plasma of knee OA subjects from New York University (NYU, $\mathrm{n=106)}$ ) and a subcohort of the phase III oral salmon calcitonin (sCT) trial SMC021-2301 (SMC, $n=147$ ). The risk of radiographic medial joint space narrowing (JSN) over 24 months was compared between quartiles (very low, low, moderate, and high) of PRO-C2. Progression was defined as two-year JSN $>0.35 \mathrm{~mm}$. Associations were adjusted for age, gender, BMI, race, baseline pain levels, and baseline joint space width.

Results: In both the NYU and SMC cohorts, subjects with low PRO-C2 levels had greater JSN compared to subjects with high PRO-C2. The mean difference in JSN between subjects with very low and high levels of PRO-C2 was $0.65 \mathrm{~mm}(\mathrm{p}=0.002)$, corresponding to a 3.4 [1.4 - 8.6]-fold higher risk of progression. There was no significant effect of SCT treatment, compared to placebo, on JSN over two years before stratification based on baseline PRO-C2. However, there were proportionately fewer progressors in the SCT arm of the very low/low PRO-C2 group compared to the moderate/high group (chi-squared $=6.5, p=$ $0.011)$.

Conclusions: Serum/plasma level of type II collagen formation, PRO-C2, may be an objective indicator of a low cartilage repair endotype, displaying radiographic progression and superior response to a proanabolic drug.

\section{Introduction}

Osteoarthritis (OA), the most common arthritis, is characterized by progressive cartilage destruction, deterioration of subchondral bone, and synovial inflammation [1, 2]. It affects nearly $10-20 \%$ of the adult population and leads to debilitating pain, functional impairment, and disability among elders [3]. OA is a highly heterogeneous disease characterized by the involvement of single or multiple joints, variable clinical features, and biochemical/genetic characteristics [4], which suggests that multiple phenotypes and endotypes exist.

A phenotype is defined as the observable properties of an organism that are produced by the interactions of the genotype and the environment. Patients with common characteristics are grouped together to guide therapy and management [5]. In contrast to a phenotype of a disease that is without any implication of a mechanism, an endotype identifies a specific biological pathway or a distinct 
pathophysiological mechanism explaining the observable characteristic of a phenotype. Endotypes are defined by specific cells or molecules in the blood, urine, and/or other biological specimens [6]. Although endotypic classification is a more specific and accurate way of defining patient subgroups, this method of classification is not yet uniformly used in the OA field [7, 8]. One simplistic and general example of a phenotype is knee pain, while the related endotype could be the synovial fluid concentration of a synovial pain marker. Determination of a disease endotype is possible when the observed changes during disease progression and/or patient response to treatment can be quantified by biomarkers [9]. Therefore, knee OA disease heterogeneity could be further explored with new biomarkers indicative of a particular endotype with distinct mechanistic pathways (e.g. low cartilage formation) and/or variable clinical presentations (e.g. radiographic fast progressors). Identification of such endotypes by validated biomarkers could assist in enabling a precision medicine approach for $\mathrm{OA}$ and eventually facilitate the development of targeted therapies for OA [7].

Recent data from the large-scale UK biobank identified genetic polymorphisms in eight genes associated with OA [10]. Three of these genes were linked to a cartilage formation/repair endotype, namely Growth Differentiation Factor 5 (GDF5), Fibroblast Growth Factor 18 (FGF18), and Transforming Growth Factorbeta 1 (TGF- $\beta 1$ ) [10], suggesting that cartilage formation, when impaired, may be associated with a higher level of OA structural disease progression due to repair attenuation. Thus, cartilage formation by chondrocytes may represent a convergent mechanism with cartilage repair pathways. By analogy to the liver fibrosis field, wherein a high fibrosis formation phenotype is more likely to respond to an anti-fibrotic therapy [11], we hypothesized that in $\mathrm{OA}$, a lower cartilage formation endotype is more likely to respond to some OA treatments.

An N-propeptidase generated biomarker of type II collagen formation in serum/plasma, the N-terminal propeptide of collagen type IIB (PIIBNP, hereafter called PRO-C2) [12], can serve as a surrogate biomarker for cartilage formation [13], making it possible to investigate a potential cartilage repair endotype in OA. We recently demonstrated that PRO-C2 concentrations were lower in OA patients compared to healthy controls and could be induced by potential cartilage anabolic therapy [14].

In the current post-hoc exploratory analysis of two independent cohorts of knee OA patients, we examined whether there was an association between baseline PRO-C2 blood levels and radiographic OA severity and progression.

\section{Methods}

\section{Study design and participants}

The NYC cohort

The New York University (NYU) cohort with varying degrees of symptomatic knee OA $(n=106)$ was recruited at New York University [15-18] (Fig. 1A). All patients underwent bilateral standardized weightbearing fixed flexion posteroanterior knee radiographs using the SynaFlexer ${ }^{\text {TM }}$ positioning frame (Synarc). 
Radiographic readings were done separately by two musculoskeletal radiologists blinded to patient information. Disagreements between the two readers were resolved by consensus. Radiographic progression was assessed by medial joint space narrowing (mJSN), based on the change in joint space width (JSW) of the signal knee at baseline and at 24 months. Medial joint space widths (mJSW) were measured at the mid-portion of the joint space via electronic calipers [15]. At baseline, all patients had Kellgren-Lawrence grade $(K L G) \geq 1$ knee OA or complained of pain in the signal knee and met American College of Rheumatology (ACR) criteria for clinical symptomatic OA. Non-fasting blood samples were collected for heparin-treated plasma and stored at $-80^{\circ} \mathrm{C}$ until biomarker measurement.

The SMC cohort

From a total of 1176 individuals in the phase III OA trial SMC021-2301 (clinicaltrial.gov: NCT00486434) testing the efficacy of oral salmon calcitonin (SCT), 200 participants recruited in one study center (Aalborg, Denmark) were selected for preliminary proof-of-concept [19, 20]. Fifty-one participants were lost at follow-up because they were missing two-year X-rays and two subjects had missing serum samples at baseline. This SMC subcohort, therefore, comprised 147 participants with knee OA (Fig. 1B). Both knees were examined during the study, but a signal knee was chosen prior to randomization based on the following inclusion criteria: KLG 2 or 3 of the medial tibiofemoral joint; a radiographic JSW of the signal knee $\geq 2.0 \mathrm{~mm}$ of the medial tibiofemoral compartment as measured on a radiograph; classification by American Rheumatism Association (ARA) criteria as Functional class I, II, or III; a Western Ontario \& McMaster Universities Osteoarthritis Index (WOMAC) version VA3.1 [21] pain subscale (five questions, a score from $0-500 \mathrm{~mm}$ ) score of $\geq 150 \mathrm{~mm}$, and/or a WOMAC function subscale (17 questions, a score from $0-1700 \mathrm{~mm}$ ) score of $\geq 510 \mathrm{~mm}$. Weight bearing knee radiographs were performed using a non-fluoroscopic, standardized, quality-controlled method as described in the previous study [19] with the SynaFlexer ${ }^{\text {TM }}$ positioning frame, and a fixed external rotation of both feet to ensure a similar standardized positioning of all patients. For a detailed description of the study population and inclusion/exclusion criteria, see $[19,20]$.

\section{Assessment of PRO-C2}

Serum or plasma PRO-C2 was assessed at baseline and 24 months in blood using a high sensitivity (hs) PRO-C2 electro-chemiluminescence ImmunoAssay (ECLIA) (Nordic Bioscience, Herlev, Denmark). It is a competitive assay based on a monoclonal antibody specific to the epitope (QDVRQPGPKG) derived from the N-terminal propeptide region of the type IIB procollagen a1-chain [14]. We have previously developed the PRO-C2 enzyme-linked immunosorbent assay (ELISA), which, had some sensitivity limitations. Therefore, we converted the assay to the ECLIA platform, resulting in a seven-fold increased sensitivity [14]. PRO-C2 was measured in the NYU cohort (non-fasting plasma) and the placebo (PLB) and sCT arms of the SMC subcohort (fasting serum) at baseline and 24 months. The effect of fasting and non-fasting was tested in 10 donor samples (Fig. S1, supplementary data). The measurement was in duplicate and blinded to the clinical data in the SMC cohort, whereas single determinations were made in the NYU cohort due to the limited volume of the samples. The intra-assay coefficient of variation (CV) of PRO-C2 was $7.4 \%$, and the inter-assay CV was $13.4 \%$. The lower limit of detection (LLOD) was $0.08 \mathrm{ng} / \mathrm{mL}$, 
defined as the concentration corresponding to 3 Standard Deviation (SD) above the mean of 21 determinations of the zero calibrator.

\section{Statistical analysis}

This is a post-hoc and explorative analysis to test the association between radiographic progression and the cartilage formation marker PRO-C2.

Baseline demographics and characteristics are presented as mean and standard deviation (SD) or as frequency and percentage (\%). Comparison of age, BMI, gender, and race between cohorts was done by either Mann-Whitney test or by chi-squared test; cohort baseline VAS pain, KLG, and mJSW were compared by multiple regression analysis adjusting for age, BMI, gender, and race.

PRO-C2 data were normalized using logarithmic transformation. Correlations with age, BMI, gender, race, with VAS pain and JSW at baseline was performed using multiple linear regression in each of the cohorts.

Each of the cohorts was dichotomized into low and high levels of PRO-C2 separated by the median using all baseline data (Fig. 1). The difference in two-year joint space narrowing (JSN) in the signal knee between low and high were analyzed in each cohort by ANCOVA with age, BMI, gender, VAS pain, and baseline medial joint space width (signal knee) as covariates. The "dose-response" association between PRO-C2 levels and JSN was investigated by first separating the subjects into quartiles (Q1 to Q4) of PRO$\mathrm{C} 2$ in the individual studies and then pooling the patients from both cohorts by quartile for purposes of assessing the association of baseline PRO-C2 with JSN. The odds ratio for progression between the PROC2 Q4 (reference) and the remaining quartiles was assessed by logistic regression, where progression was defined as $\mathrm{JSN}>0$ (any progression). The analysis was adjusted for the covariates.

Proportional difference in responders and non-responders between placebo and SCT arms of the SMC study in groups of low and high (median cut-off) levels of baseline PRO-C2 was tested by chi-square. Non-responders were defined as those with JSN $>0.35 \mathrm{~mm}$.

All the statistical analyses were performed with MedCalc version 19.1.7 (MedCalc Software, Ostend, Belgium), and graphing was done with GraphPad Prism version 8.3 (GraphPad Software, CA, USA). The significance level was set at $a=0.05$, but reported to 0.1

\section{Results}

\section{Study populations and baseline characteristics}

From 147 enrolled in the NYC study, 31 were lost at follow-up and ten patients were excluded due to the lack of plasma samples. The remaining 106 individuals were dichotomized according to the median level of baseline plasma PRO-C2 (Fig. 1A). The 147 subjects in the sSMC subcohort were dichotomized based on the median level of baseline serum PRO-C2 (Fig. 1B). 
Demographic, clinical, and radiographic details of the 106 NYC and the 147 SMC participants are summarized in Table 1. Mean age and BMI were higher in the SMC cohort compared to the NYC cohort. There were more female participants in the NYC cohort, but not significantly. There were only Caucasians in the SMC cohort in contrast to the NYC cohort where there were $66 \%$ Caucasians, $25 \%$ blacks, and $9 \%$ other. Approximately $21 \%$ of the NYC participants used NSAIDs at baseline, whereas none of the SMC participants did.

Table 1

Cohort description

\begin{tabular}{|c|c|c|c|c|}
\hline \multicolumn{2}{|l|}{ Variables } & $\begin{array}{l}\text { NYU cohort }(N= \\
106)\end{array}$ & $\begin{array}{l}\text { SMC cohort }(N \\
=147)\end{array}$ & $\begin{array}{l}\text { Difference between cohorts } \\
\text { ( } p \text { value) }\end{array}$ \\
\hline \multicolumn{2}{|c|}{ Age, mean (SD) years } & $61.0(10.2)$ & $63.6(6.6)$ & 0.026 \\
\hline \multicolumn{2}{|c|}{ BMI, mean (SD) kg/m² } & $26.6(3.6)$ & $29.0(4.5)$ & 0.0007 \\
\hline \multicolumn{2}{|c|}{ Gender, no. female (\%) } & $69(65)$ & $82(56)$ & ns \\
\hline \multicolumn{2}{|c|}{$\begin{array}{l}\text { Race, no. white/black / other } \\
(\%)\end{array}$} & $\begin{array}{l}70(66) / 26(25) / \\
10(9)\end{array}$ & $\begin{array}{l}147(100) / 0 / \\
0\end{array}$ & $<0.0001$ \\
\hline \multicolumn{2}{|c|}{$\begin{array}{l}\text { Pain, mean (SE) VAS 0- } \\
100 \mathrm{~mm}\end{array}$} & $42.1(2.7)$ & $48.2(2.1)$ & $\mathrm{ns}^{1}$ \\
\hline \multicolumn{2}{|c|}{$\begin{array}{l}\text { mJSW on signal knee, mean } \\
\text { (SE) mm }\end{array}$} & $3.63(0.10)$ & $3.31(0.09)$ & $0.022^{1}$ \\
\hline \multirow[t]{3}{*}{ KLG, Frequency (\%) } & $0-1$ & $29(27)$ & 0 & $0.030^{1}$ \\
\hline & 2 & $20(19)$ & $129(88)$ & \\
\hline & $3-4$ & $57(54)$ & $18(12)$ & \\
\hline \multicolumn{5}{|c|}{$\begin{array}{l}\text { * Normalized to } 500 \mathrm{~mm} \text { score scale. † normalized to } 1700 \mathrm{~mm} \text { score scale. BMI, body mass index; } \\
\text { KLG, Kellgren-Lawrence grades; mJSW, medial joint space width; ns, not significant; SD, standard } \\
\text { deviation; SE, standard error; VAS, visual analog scale; WOMAC, Western Ontario \& McMaster } \\
\text { Universities Osteoarthritis Index. }\end{array}$} \\
\hline
\end{tabular}

After adjusting for age, gender, race, and $\mathrm{BMI}$, there was no difference in the average pain score of the signal knee between the two cohorts (Table 1). Mean JSW of the signal knee was significantly higher in the NYC cohort after adjusting for age, BMI, gender, and race. The NYC cohort included patients with KLG 0 to 4 in the signal knee, whereas the SMC cohort only included patients with KLG 2 and 3 (Table 1).

Serum/plasma PRO-C2 levels were correlated with age $(r=0.41, p=0.0001)$ and race $(r=0.34, p=0.0010)$ in the NYC cohort and with BMI $(r=0.24, p=0.0013)$ and JSW $(r=-0.54, p<0.0001)$ in the SMC cohort in univariate testing (Table 2). 
Table 2

Correlation (multiple linear regression) between PRO-C2 and baseline characteristics. Data are shown as partial $r$ ( $p$ value), where non-significant ( $n s)$ is $p>0.1$. BMI: Body mass index; $m J S W$, medial joint space width; ns, not significant, VAS, visual analogue scale.

\begin{tabular}{|lllllll|}
\hline & Age & BMI & Gender & Race & Pain VAS* & mJSW* \\
\hline NYC & $0.41(0.0001)$ & $0.19(n s)$ & $0.17(n s)$ & $0.34(0.0010)$ & $0.06(n s)$ & $0.20(n s)$ \\
\hline SMC & $-0.10(n s)$ & $0.24(0.0013)$ & $0.11(n s)$ & - & $-0.09(n s)$ & $-0.54(<0.0001)$ \\
\hline
\end{tabular}

\section{Association between baseline PRO-C2 and medial joint space narrowing}

Difference in two-year JSN was assessed in the NYC and SMC cohorts on the dichotomized data (Fig. 1). Patients with low PRO-C2 progressed significantly faster than patients with high PRO-C2 in the NYC cohort: mean difference was $0.52 \mathrm{~mm}(p=0.0078)$ (Fig. 2A). Similarly, in the SMC cohort, JSN was greater in the low PRO-C2 group compared to the higher PRO-C2 group of the placebo arm: mean difference was $0.24(p=0.078)$ (Fig. $2 B)$, however, this difference was of borderline significance. Scatter plots of the PRO-C2 distribution and summary statistics for each of the subgroups can be found in the supplementary data file (Fig. S2).

Next we pooled the data of the two cohorts to assess the overall "dose-response" of JSN as a function of baseline PRO-C2 levels. The mean differences between the lowest level of PRO-C2 (Q1, very low) and the higher levels of PRO-C2 were: Q2 (low), 0.30 (ns); Q3 (moderate), 0.48 ( $p=0.056)$; and Q4 (high), 0.65 ( $p=$ 0.0018) (Fig. 2C). These data indicate that radiographic progression is more pronounced in patients with low type II collagen formation, an effect that was independent of the covariates. Scatter plots of the PROC2 distribution can be found in the supplementary data file (Fig. S2).

\section{Subjects with very low levels of baseline PRO-C2 are more likely to progress}

We investigated the odds ratio (OR) for progression of the different groups using the high PRO-C2 group (Q4 in Fig. 2) as a reference. The participants in the very low group (Q1) were more likely to progress over the two-year period than the high group: OR 3.4 [1.4-8.6], $p=0.0087$ (Fig. 3). A similar likelihood of progression was observed in the very low PRO-C2 group of participants with definite radiographic OA ( $K L G \geq 2)$ : OR 3.9 [1.4-11.3], $p=0.011$ (Fig. 3). Subjects with low (Q2) and moderate (Q3) levels were not more likely to progress compared with those with high levels (Q4) (Fig. 3).

\section{PRO-C2 levels and treatment response}

First we investigated whether there was a proportional difference in the number of responders and nonresponders to oral salmon calcitonin in low and high PRO-C2 subgroups. There were proportionally fewer non-responders in the low PRO-C2 - sCT arm (Chi-squared of 6.5, $p=0.011$ ) (Table 3). 
Table 3

Proportion of responders and non-responders in high and low baseline PRO-C2 groups. Non-responders were defined as those with $\mathrm{JSN}>0.35 \mathrm{~mm}$. Data are shown as number of subjects (\%). JSN, joint space narrowing; SCT, salmon

calcitonin.

\begin{tabular}{|c|c|c|c|c|}
\hline & & $\begin{array}{l}\text { Responders } \\
\text { JSN } \leq 0 \mathrm{~mm}\end{array}$ & $\begin{array}{l}\text { Intermediate responders } \\
0<\mathrm{JSN} \leq 0.35 \mathrm{~mm}\end{array}$ & $\begin{array}{l}\text { Non-responders } \\
\text { JSN }>0.35 \mathrm{~mm}\end{array}$ \\
\hline \multirow[t]{2}{*}{ Low } & Placebo & $13(33)$ & $5(13)$ & $22(55)$ \\
\hline & $\mathrm{sCT}$ & $14(45)$ & $8(26)$ & 9 (29) \\
\hline \multirow[t]{2}{*}{ High } & Placebo & $17(44)$ & $10(26)$ & $12(31)$ \\
\hline & $\mathrm{sCT}$ & $14(38)$ & $9(24)$ & $14(38)$ \\
\hline
\end{tabular}

Lastly we assessed the pharmacodynamic effect of SCT vs. placebo in either low or high PRO-C2 groups. In patients with low baseline PRO-C2, there was an approximate $20 \%$ increase in PRO-C2 levels in patients treated with SCT; however, this was not significantly different from the increase (approximately 15\%) observed in the placebo (Fig. 4). Both treatment arms showed decreasing levels in PRO-C2 of approximately $15 \%$ (Fig. 4).

\section{Discussion}

Type II collagen is nearly exclusively localized to cartilage, being the major structural component of this tissue. Recently, immunoassays suitable for measuring serological collagen type IIB N-propeptide fragment (PIIBNP or PRO-C2) were developed and proposed for the estimation of cartilage formation [14]. In the present study, we investigated the clinical utility of PRO-C2. Low baseline PRO-C2 identified a more rapidly progressing subgroup in two independent knee OA clinical cohorts. This suggests that low cartilage formation may be associated with an endotype of higher structural loss. These data agree with results from the Foundation for National Institute of Health (FNIH) initiative on PIIANP [22], another type II collagen biomarker indicative of collagen formation. In addition, cartilage oligomeric protein (COMP) and urinary CTX-II (UCTX-II) have all shown potential as prognostic biomarkers [22-27]. Interestingly, however, UCTX-II and PIIANP did not provide the same prognostic, nor predictive value in the current study (see supplementary data Fig. S4), and COMP was not investigated. Nevertheless, the fact that PRO-C2 and several other biomarkers, all indicative of joint tissue turnover, are able to predict progression supports the potential to identify one or more clinically relevant endotypes of OA using molecular markers. This is the long-lost promise of precision medicine. Although several initiatives are currently underway to qualify molecular biomarkers for use in OA trials, such as the APPROACH (Applied Public-Private Research enabling OsteoArthritis Clinical Headway) consortium and the FNIH initiative on soluble biomarkers for $\mathrm{OA}$, there is still an urgent need to develop novel and sensitive biomarkers that could be used for categorizing patients, trial enrichment, and personalized healthcare in OA [28, 29]. 
In the osteoporosis field, it is well-known that bone turnover activity is positively associated with the level of response to treatment [30]. In other words, patients with osteoporosis and a high bone turnover respond better to anti-resorptive (anti-catabolic) [30] and pro-formation (anabolic) therapies [31]. Additionally, patients with high fibrogenic activity or fibrosis formation (namely rapid progressors) are more likely to respond to an anti-fibrotic therapy in the liver fibrosis [11]. By analogy to both diseases, a similar approach and paradigm may be applicable to $O A$ and assist in the path towards developing a more endotype specific approach OA therapy, thereby allowing for personalized musculoskeletal health care. Biomarkers related to disease progression and response to a selected intervention would greatly aid the success of drug development in OA. Participants with lower baseline PRO-C2 had a better response to treatment with $\mathrm{SCT}$, as measured by JSN, compared to the placebo group. This suggests that PRO-C2 could serve as a biomarker not only for prediction of OA progression but also for treatment response. We recently demonstrated that FGF-18 induced PRO-C2 in both human and animal chondrocytes [14, 32]. In addition, in the phase II FORWARD (FGF-18 Osteoarthritis Randomized Trial with Administration of Repeated Doses) study, intra-articular FGF-18 demonstrated a dose-dependent increase in the cartilage thickness on Magnetic Resonance Imaging (MRI) [33, 34]. In this trial, synovial fluid PRO-C2 levels increased over time in OA patients treated with FGF18, whereas no change was observed in the placebo arm (unpublished data). Moreover, there were notable differences of cartilage thickness change over 2years in the total femorotibial joint between OA patients with lower levels of PRO-C2 at baseline and those with high PRO-C2. Compared with the high PRO-C2 subgroup, the low PRO-C2 subgroup demonstrated greater increase in total cartilage thickness and improved outcomes for WOMAC total scores after 2 years of treatment of FGF18 compared to the placebo group [35]. These combined data suggest a causal relationship between PRO-C2 and cartilage formation [36]. It may be possible to extend the treatment response predictability of PRO-C2 to other DMOADs.

$\mathrm{OA}$ is a heterogeneous disease, and multiple phenotypes of $\mathrm{OA}$ have been identified based on risk factors such as aging, metabolic syndrome, trauma, endocrine, inflammatory, and subchondral bone-driven progression of OA [37-40]. Some corresponding molecular and/or cellular endotypes have also been reported (Fig. 5), although the terms "endotype" and "phenotype" have been used in confusing ways in different reports. For instance, recent work by $\mathrm{Ji}$ et al. investigated the relationships between seven endotypes of OA cartilage chondrocytes and OA severity $[41,42]$. They stated that the subgroups of proliferative chondrocytes, pre-hypertrophic chondrocytes, and fibrocartilage chondrocytes were correlated with worse clinical outcomes. The study by Zhang WD et al. identified two metabolic endotypes of knee osteoarthritis that differed in synovial fluid concentrations of acylcarnitine and carnitine [43], which would help to unravel the pathogenesis and develop targeted therapies for OA. In a recent study, glucosepane markedly increased with age and disease progression in both a guinea pig model of knee $O A$ and osteoarthritis in patients [44]. The association of glucosepane with aging-related OA may, for the first time, improve early-stage OA diagnosis and prognosis. Additionally, Huebner et al. observed that a high bone absorption endotype (measured by alpha CTX-I) was associated with OA progression defined by features of JSN and osteophyte in a longitudinal study of patients with symptomatic OA [45]. This indicates that OA treatment should include the targeting of the subchondral 
bone. In an inflammatory OA phenotype, six synovial fluid biomarkers were recently reported to be specific indicators of an endotype characterized by activated macrophages and neutrophils [46]. In the present study, we discovered a low cartilage formation endotype (measured by the type II collagen formation biomarker, PRO-C2) corresponding to a cartilage-driven phenotype presenting with a higher level of disease progression and superior response to a potential OA treatment. All in all, the progress of understanding the meaning of molecular endotypes and clinical phenotypes could help shed light on the pathophysiological mechanism of $O A$ and aid in patient stratification, better design of clinical trials, and personalized treatments for knee OA patients.

There are several limitations associated with the current study. First, the cut-off values, based upon the median concentrations in the two cohorts, SMC and NYU, were different; this was due to different levels in serum and plasma but also a consequence of distinct clinical characteristics of the two cohorts. The difference seemed to have nothing to do with the fasting vs non-fasting sampling, in that there was no significant difference in PRO-C2 levels between fasting and non-fasting blood from healthy individuals (see supplementary data Fig. S1). Further validation in larger populations will be needed to determine the specific optimal threshold values for distinguishing OA progression risk. Second, the durations of follow-

up for both NYU and SMC cohorts were only two years; hence, it remains unknown whether PRO-C2 would predict progression over a longer follow-up period. Third, the number of participants investigated in the longitudinal assessment was relatively small; larger populations (e.g., the entire SMC021-2301 cohort) are required to confirm these findings. Fourth, further studies comparing serum and synovial fluid concentrations of PRO-C2 in well-characterized OA cohorts are warranted to reveal the clinical significance of PRO-C2 considering the fact that synovial fluid is subject to less interference from systemic sources of noise. Fifth, we used only radiographic medial JSW, not MRI, as a surrogate marker for evaluating cartilage degradation of OA progression, as both cohorts were designed one decade ago when MRI features and lateral JSW were not commonly assessed in trials. Lastly, this study examined only knee OA patients, and therefore the results may not be generalizable to patients with other types of OA, e.g., hand and/or hip OA.

\section{Conclusion}

In conclusion, these data suggest that endotyping in knee OA patients is feasible. We report that low cartilage formation based on PRO-C2 appears to be a quantifiable OA endotype associated with structural OA progression and response to treatment.

\section{List Of Abbreviations}

ACR: American College of Rheumatology; ARA: American Rheumatism Association; ARGS: N-terminal neoepitope of the aggrecanase-mediated aggrecan degradation fragment; BMI: Body mass index; C1M: MMP-derived collagen degradation neoepitopes of collagen I; C3M: MMP-derived collagen degradation neoepitopes of collagen III; COMP: cartilage oligomeric protein; CRPM: MMP-depended degradation product of C-reactive protein; CTX-I: C-terminal telopeptide of collagen I; CV: coefficient of variation; 
DMOAD: Disease-modifying osteoarthritis drug; ECLIA: Electrochemiluminescence immunoassay; ECM: Extracellular matrix; FGF18: Fibroblast Growth Factor 18; GDF5: Growth Differentiation Factor 5; IL-6: interleukin-6; IL-17: interleukin-17; KLG: Kellgren-Lawrence grades; LLOD: Lower limit of detection; LLOQ: lower limit of quantification; MCP-1: monocyte chemoattractant protein 1; mJSW: medial joint space width; MMP-3: matrix metalloproteinase-3; MRI: Magnetic resonance imaging; ns: not significant; OA: Osteoarthritis; PLB: placebo; PIIANP: Procollagen type IIA N-terminal propeptides; PIIBNP (N-terminal propeptide of procollagens IIB); SCT: salmon calcitonin; SD: standard deviation; SE: standard error; SEM: Standard error of mean; sICAM-1: soluble intracellular adhesion molecule 1; sVCAM-1: soluble vascular cell adhesion molecule 1; TGF- $\beta 1$ : Transforming Growth Factor-beta 1; TNF-a: tumour necrosis factoralpha; TIMP-1: tissue inhibitor of metallopeptidase inhibitor 1; VAS: visual analogue scale; VEGF: vascular endothelial growth factor; WOMAC: Western Ontario \& McMaster Universities Osteoarthritis Index.

\section{Declarations}

\section{Ethics approval and consent to participate}

Approval for the study was obtained from the institutional ethics review committee at NYU and the CCBR Aalborg study centre.

\section{Consent for publication}

Not applicable

\section{Availability of data and materials}

The datasets used and/or analysed during the current study are available from the corresponding author on reasonable request.

\section{Competing interests}

$\mathrm{YH}, \mathrm{IB}, \mathrm{MAK}$ and ACBJ are full-time employees, and the latter two are shareholders of Nordic Bioscience. $\mathrm{YL}$ are associated Nordic Bioscience as visiting scientist, however, employed by University of Copenhagen.

\section{Funding}

The NYU study was supported by National Institute of Health, National Institute of Arthritis and Musculoskeletal and Skin Diseases: R01 AR052873 (to SBA). The funding sources had no role in study design, in collection, analysis or interpretation of data, or in writing the manuscript and decision to submit the manuscript.

\section{Author contributions}


ACBJ, MA, MK and IB designed the study and contributed to the discussions; JS, SK, IB, SBA MAK and MA were responsible for clinical data acquition; $\mathrm{YL}, \mathrm{YH}$ and $\mathrm{ACBJ}$ carried out the biomarker measurement, data analysis and drafted the manuscript; YL, YH, ACBJ, VBK, MK, SBA, and MA were major contributors in writing the manuscript. All authors commented, reviewed and approved the final manuscript for submission.

\section{Acknowledgments}

The authors would like to thank Niamh Higgins for sorting out serum samples in the SMC subcohort. This study was financially supported by the Danish Research Foundation (Den Danske Forskningsfond).

\section{References}

[1] F. Berenbaum, Osteoarthritis as an inflammatory disease (osteoarthritis is not osteoarthrosis!), Osteoarthr. Cartil. (2013). doi:10.1016/j.joca.2012.11.012.

[2] S.N. Issa, L. Sharma, Epidemiology of osteoarthritis: an update., Curr. Rheumatol. Rep. 8 (2006) 715. http://www.ncbi.nlm.nih.gov/pubmed/16515759 (accessed April 4, 2019).

[3] D.T. Felson, Identifying different osteoarthritis phenotypes through epidemiology, Osteoarthr. Cartil. (2010). doi:10.1016/j.joca.2010.01.007.

[4] J.B. Driban, M.R. Sitler, M.F. Barbe, E. Balasubramanian, Is osteoarthritis a heterogeneous disease that can be stratified into subsets?, Clin. Rheumatol. (2010). doi:10.1007/s10067-009-1301-1.

[5] S.E. Wenzel, Asthma phenotypes: The evolution from clinical to molecular approaches, Nat. Med. (2012). doi:10.1038/nm.2678.

[6] M.E. Kuruvilla, F.E.H. Lee, G.B. Lee, Understanding Asthma Phenotypes, Endotypes, and Mechanisms of Disease, Clin. Rev. Allergy Immunol. (2019). doi:10.1007/s12016-018-8712-1.

[7] A. Mobasheri, W.E. van Spil, E. Budd, I. Uzieliene, E. Bernotiene, A.C. Bay-Jensen, J. Larkin, M.C. Levesque, O. Gualillo, Y. Henrotin, Molecular taxonomy of osteoarthritis for patient stratification, disease management and drug development: biochemical markers associated with emerging clinical phenotypes and molecular endotypes, Curr. Opin. Rheumatol. 31 (2019) 80-89.

doi:10.1097/BOR.0000000000000567.

[8] S.M. Bierma-Zeinstra, M. van Middelkoop, In search of phenotypes, Nat. Rev. Rheumatol. 13 (2017) 705-706. doi:10.1038/nrrheum.2017.181.

[9] M. Jutel, P. Gajdanowicz, Implementing Precision Medicine in Best Practices of Chronic Airway Diseases: Chapter 5 - Revised Disease Nomenclature Including Disease Endotypes, Academic Press, 2019. doi:10.1016/B978-0-12-813471-9.00029-3. 
[10] I. Tachmazidou, K. Hatzikotoulas, L. Southam, J. Esparza-Gordillo, V. Haberland, J. Zheng, T. Johnson, M. Koprulu, E. Zengini, J. Steinberg, J.M. Wilkinson, S. Bhatnagar, J.D. Hoffman, N. Buchan, D. Süveges, L. Yerges-Armstrong, G.D. Smith, T.R. Gaunt, R.A. Scott, L.C. McCarthy, E. Zeggini, Identification of new therapeutic targets for osteoarthritis through genome-wide analyses of UK Biobank data, Nat. Genet. (2019). doi:10.1038/s41588-018-0327-1.

[11] M.A. Karsdal, K. Henriksen, M.J. Nielsen, I. Byrjalsen, D.J. Leeming, S. Gardner, Z. Goodman, K. Patel, A. Krag, C. Christiansen, D. Schuppan, Fibrogenesis assessed by serological type III collagen formation identifies patients with progressive liver fibrosis and responders to a potential antifibrotic therapy., Am. J. Physiol. Gastrointest. Liver Physiol. (2016). doi:10.1152/ajpgi.00283.2016.

[12] R.J. Fernandes, S. Hirohata, J.M. Engle, A. Colige, D.H. Cohn, D.R. Eyre, S.S. Apte, Procollagen II amino propeptide processing by ADAMTS-3. Insights on dermatosparaxis, J. Biol. Chem. 276 (2001) 31502-31509. doi:10.1074/jbc.M103466200.

[13] N. Gudmann, J. Wang, S. Hoielt, P. Chen, A. Siebuhr, Y. He, T. Christiansen, M. Karsdal, A. BayJensen, Cartilage Turnover Reflected by Metabolic Processing of Type II Collagen: A Novel Marker of Anabolic Function in Chondrocytes, Int. J. Mol. Sci. 15 (2014) 18789-18803. doi:10.3390/ijms151018789.

[14] Y. Luo, Y. He, D. Reker, N.S. Gudmann, K. Henriksen, A Novel High Sensitivity Type II Collagen BloodBased Biomarker, PRO-C2, for Assessment of Cartilage Formation, Int. J. Mol. Sci. 19 (2018) 3485. doi:10.3390/ijms19113485.

[15] M. Attur, I. Belitskaya-lévy, C. Oh, S. Lee, J. Patel, H. Al-mussawir, G. Mcdaniel, Increased IL-1 beta gene expression in peripheral blood leukocytes is associated with increased pain and predicts risk for progression of symptomatic knee osteoarthritis, Arthritis Rheumatol. 63 (2011) 1908-1917. doi:10.1002/art.30360.Increased.

[16] M. Attur, A. Statnikov, J. Samuels, Z. Li, A. V. Alekseyenko, J.D. Greenberg, S. Krasnokutsky, L. Rybak, Q.A. Lu, J. Todd, H. Zhou, J.M. Jordan, V.B. Kraus, C.F. Aliferis, S.B. Abramson, Plasma levels of interleukin-1 receptor antagonist (IL1Ra) predict radiographic progression of symptomatic knee osteoarthritis, Osteoarthr. Cartil. 23 (2015) 1915-1924. doi:10.1016/j.joca.2015.08.006.

[17] S. Krasnokutsky, C. Oshinsky, M. Attur, S. Ma, H. Zhou, F. Zheng, M. Chen, J. Patel, J. Samuels, V.C. Pike, R. Regatte, J. Bencardino, L. Rybak, S. Abramson, M.H. Pillinger, Serum Urate Levels Predict Joint Space Narrowing in Non-Gout Patients With Medial Knee Osteoarthritis, Arthritis Rheumatol. 69 (2017) 1213-1220. doi:10.1002/art.40069.

[18] E. Bournazou, J. Samuels, H. Zhou, S. Krasnokutsky, J. Patel, T. Han, J. Bencardino, L. Rybak, S.B. Abramson, U. Junker, K.S. Brown, M. Attur, Vascular Adhesion Protein-1 (VAP-1) as Predictor of Radiographic Severity in Symptomatic Knee Osteoarthritis in the New York University Cohort, Int. J. Mol. Sci. 20 (2019) 2642. doi:10.3390/ijms20112642. 
[19] M. a. Karsdal, I. Byrjalsen, P. Alexandersen, a. Bihlet, J.R. Andersen, B.J. Riis, a. C. Bay-Jensen, C. Christiansen, Treatment of symptomatic knee osteoarthritis with oral salmon calcitonin: results from two phase 3 trials, Osteoarthr. Cartil. 23 (2015) 532-543. doi:10.1016/j.joca.2014.12.019.

[20] M.A. Karsdal, M. Michaelis, C. Ladel, A.S. Siebuhr, A.R. Bihlet, J.R. Andersen, H. Guehring, C. Christiansen, A.C. Bay-Jensen, V.B. Kraus, Disease-modifying treatments for osteoarthritis (DMOADs) of the knee and hip: lessons learned from failures and opportunities for the future, Osteoarthr. Cartil. (2016) 1-9. doi:10.1016/j.joca.2016.07.017.

[21] N. Bellamy, W.W. Buchanan, C.H. Goldsmith, J. Campbell, L.W. Stitt, Validation study of WOMAC: A health status instrument for measuring clinically important patient relevant outcomes to antirheumatic drug therapy in patients with osteoarthritis of the hip or knee, J. Rheumatol. (1988).

[22] V.B. Kraus, J.E. Collins, D. Hargrove, E. Losina, M. Nevitt, J.N. Katz, S.X. Wang, L.J. Sandell, S.C. Hoffmann, D.J. Hunter, Predictive validity of biochemical biomarkers in knee osteoarthritis: data from the FNIH OA Biomarkers Consortium, Ann. Rheum. Dis. 76 (2017) 186-195. doi:10.1136/annrheumdis-2016209252.

[23] P. Garnero, X. Ayral, J.-C. Rousseau, S. Christgau, L.J. Sandell, M. Dougados, P.D. Delmas, Uncoupling of type II collagen synthesis and degradation predicts progression of joint damage in patients with knee osteoarthritis, Arthritis Rheum. 46 (2002) 2613-2624. doi:10.1002/art.10576.

[24] M. Sharif, J. Kirwan, N. Charni, L.J. Sandell, C. Whittles, P. Garnero, A 5-yr longitudinal study of type IIA collagen synthesis and total type II collagen degradation in patients with knee osteoarthritisassociation with disease progression., Rheumatology (Oxford). 46 (2007) 938-943. doi:10.1093/rheumatology/kel409.

[25] a. M. Valdes, I. Meulenbelt, E. Chassaing, N.K. Arden, S. Bierma-Zeinstra, D. Hart, a. Hofman, M. Karsdal, M. Kloppenburg, H.M. Kroon, E.P. Slagboom, T.D. Spector, a. G. Uitterlinden, J.B. Van Meurs, a. C. Bay-Jensen, Large scale meta-analysis of urinary C-terminal telopeptide, serum cartilage oligomeric protein and matrix metalloprotease degraded type II collagen and their role in prevalence, incidence and progression of osteoarthritis, Osteoarthr. Cartil. 22 (2014) 683-689. doi:10.1016/j.joca.2014.02.007.

[26] A. Struglics, S. Larsson, N. Kumahashi, R. Frobell, L.S. Lohmander, Changes in Cytokines and Aggrecan ARGS Neoepitope in Synovial Fluid and Serum and in C-Terminal Crosslinking Telopeptide of Type II Collagen and N-Terminal Crosslinking Telopeptide of Type I Collagen in Urine Over Five Years After Anterior Cruciate Ligame, Arthritis Rheumatol. 67 (2015) 1816-1825. doi:10.1002/art.39146.

[27] L.F. Schaefer, M. Sury, M. Yin, S. Jamieson, I. Donnell, S.E. Smith, J.A. Lynch, M.C. Nevitt, J. Duryea, Quantitative measurement of medial femoral knee cartilage volume - analysis of the OA Biomarkers Consortium FNIH Study cohort, Osteoarthr. Cartil. (2017). doi:10.1016/j.joca.2017.01.010. 
[28] V.B. Kraus, F.J. Blanco, M. Englund, M. a. Karsdal, L.S. Lohmander, Call for standardized definitions of osteoarthritis and risk stratification for clinical trials and clinical use, Osteoarthr. Cartil. (2015) 1-9. doi:10.1016/j.joca.2015.03.036.

[29] M.A. Karsdal, C. Christiansen, C. Ladel, K. Henriksen, V.B. Kraus, A.C. Bay-Jensen, Osteoarthritis - a case for personalized health care?, Osteoarthr. Cartil. 22 (2014) 7-16. doi:10.1016/j.joca.2013.10.018.

[30] J.P. Lee, E. Murray, A.J. Bennett, D.J.P. Ellis, Response of Bone Turnover Markers to Three Oral Bisphosphonate Therapies in Postmenopausal Osteoporosis: the TRIO Study, Osteoporos Int. 27 (2016) 21-31.

[31] L.A. Fitzpatrick, R. Eastell, M. Hu, D.M. Black, Y. Wang, B.H. Mitlak, Bone turnover markers to explain changes in lumbar spine BMD with abaloparatide and teriparatide: results from ACTIVE, Osteoporos. Int. (2019) 667-673. doi:10.1007/s00198-018-04819-1.

[32] D. Reker, C.F. Kjelgaard-Petersen, A.S. Siebuhr, M. Michaelis, A. Gigout, M.A. Karsdal, C. Ladel, A.C. Bay-Jensen, Sprifermin (rhFGF18) modulates extracellular matrix turnover in cartilage explants ex vivo, J. Transl. Med. 15 (2017) 1-12. doi:10.1186/s12967-017-1356-8.

[33] F. Eckstein, S. Wax, A. Aydemir, W. Wirth, S. Maschek, M. Hochberg, Intra-articular sprifermin reduces cartilage loss in addition to increasing cartilage gain independent of femorotibial location: a post-hoc analysis of a randomized, placebo-controlled phase ii clinical trial, Osteoarthr. Cartil. (2018). doi:10.1016/j.joca.2018.02.590.

[34] M.C. Hochberg, A. Guermazi, H. Guehring, A. Aydemir, S. Wax, P. Fleuranceau-Morel, A. Reinstrup Bihlet, I. Byrjalsen, J. Ragnar Andersen, F. Eckstein, Effect of Intra-Articular Sprifermin vs Placebo on Femorotibial Joint Cartilage Thickness in Patients With Osteoarthritis, J. Am. Med. Assoc. 322 (2019) 1360. doi:10.1001/jama.2019.14735.

[35] C. Ladel, H. Guehring, A.-C. Bay-Jensen, M. Karsdal, P. Qvist, Metabolic biomarkers for predicting responsiveness to FGF-18 compound, WO 2019/063758 Al, 2019.

[36] F. Eckstein, W. Wirth, A. Guermazi, S. Maschek, A. Aydemir, Brief Report: Intraarticular Sprifermin Not Only Increases Cartilage Thickness, but Also Reduces Cartilage Loss: Location-Independent Post Hoc Analysis Using Magnetic Resonance Imaging, Arthritis Rheumatol. 67 (2015) 2916-2922. doi:10.1002/art.39265.

[37] M. Van der Esch, J. Knoop, M. van der Leeden, L.D. Roorda, W.F. Lems, D.L. Knol, J. Dekker, Clinical phenotypes in patients with knee osteoarthritis: A study in the Amsterdam osteoarthritis cohort, Osteoarthr. Cartil. (2015). doi:10.1016/j.joca.2015.01.006.

[38] M. a. Karsdal, a. Bihlet, I. Byrjalsen, P. Alexandersen, C. Ladel, M. Michaels, J.R. Andersen, B.J. Riis, V. Kraus, a. C. Bay-Jensen, C. Christiansen, OA phenotypes, rather than disease stage, drive structural 
progression - identification of structural progressors from 2 phase III randomized clinical studies with symptomatic knee OA, Osteoarthr. Cartil. 23 (2015) 550-558. doi:10.1016/j.joca.2014.12.024.

[39] A. Dell'Isola, R. Allan, S.L. Smith, S.S.P. Marreiros, M. Steultjens, Identification of clinical phenotypes in knee osteoarthritis: a systematic review of the literature, BMC Musculoskelet. Disord. (2016). doi:10.1186/s12891-016-1286-2.

[40] A. Mobasheri, M.P. Rayman, O. Gualillo, J. Sellam, P. Van Der Kraan, U. Fearon, The role of metabolism in the pathogenesis of osteoarthritis, Nat. Rev. Rheumatol. (2017). doi:10.1038/nrrheum.2017.50.

[41] F. Berenbaum, Deep phenotyping of osteoarthritis: A step forward, Ann. Rheum. Dis. 78 (2019) 3-5. doi:10.1136/annrheumdis-2018-213864.

[42] Q. Ji, Y. Zheng, G. Zhang, Y. Hu, X. Fan, Y. Hou, L. Wen, L. Li, Y. Xu, Y. Wang, F. Tang, Single-cell RNAseq analysis reveals the progression of human osteoarthritis, Ann. Rheum. Dis. (2019). doi:10.1136/annrheumdis-2017-212863.

[43] W. Zhang, S. Likhodii, Y. Zhang, E. Aref-Eshghi, P.E. Harper, E. Randell, R. Green, G. Martin, A. Furey, G. Sun, P. Rahman, G. Zhai, Classification of osteoarthritis phenotypes by metabolomics analysis., BMJ Open. 4 (2014) e006286. doi:10.1136/bmjopen-2014-006286.

[44] C. Legrand, U. Ahmed, A. Anwar, K. Rajpoot, S. Pasha, C. Lambert, R.K. Davidson, I.M. Clark, P.J. Thornalley, Y. Henrotin, N. Rabbani, Glycation marker glucosepane increases with the progression of osteoarthritis and correlates with morphological and functional changes of cartilage in vivo, Arthritis Res. Ther. (2018). doi:10.1186/s13075-018-1636-6.

[45] J.L. Huebner, A.C. Bay-Jensen, K.M. Huffman, Y. He, D.J. Leeming, G.E. McDaniel, M.A. Karsdal, V.B. Kraus, Alpha C-telopeptide of type I collagen is associated with subchondral bone turnover and predicts progression of joint space narrowing and osteophytes in osteoarthritis., Arthritis Rheumatol. (Hoboken, N.J.). 66 (2014) 2440-2449. doi:10.1002/art.38739.

[46] C.A. Haraden, J.L. Huebner, M.F. Hsueh, Y.J. Li, V.B. Kraus, Synovial fluid biomarkers associated with osteoarthritis severity reflect macrophage and neutrophil related inflammation, Arthritis Res. Ther. (2019). doi:10.1186/s13075-019-1923-x.

\section{Figures}


A

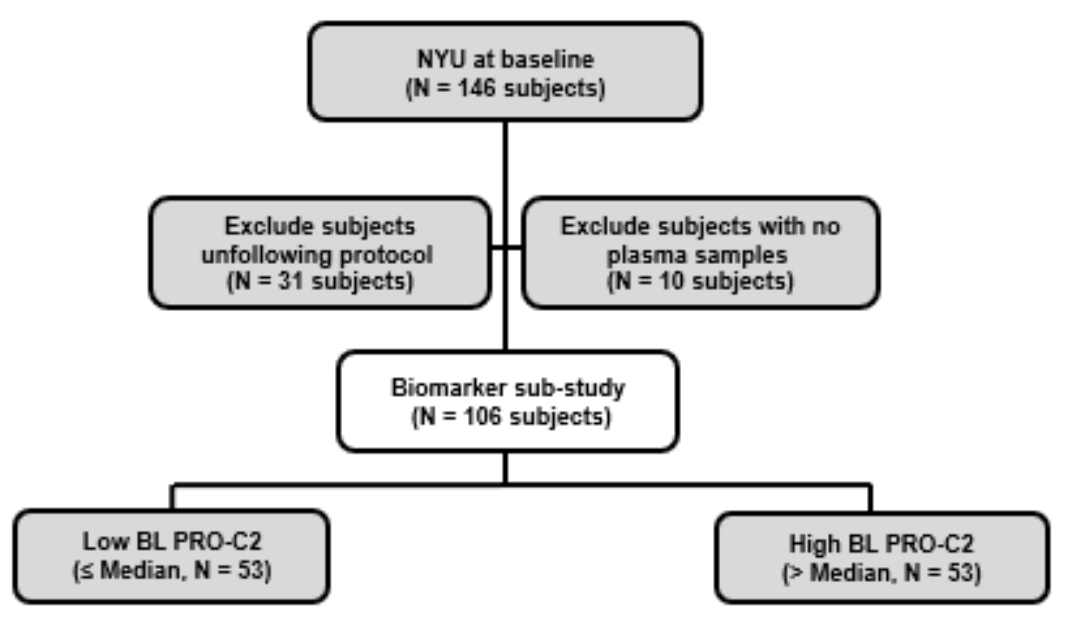

B
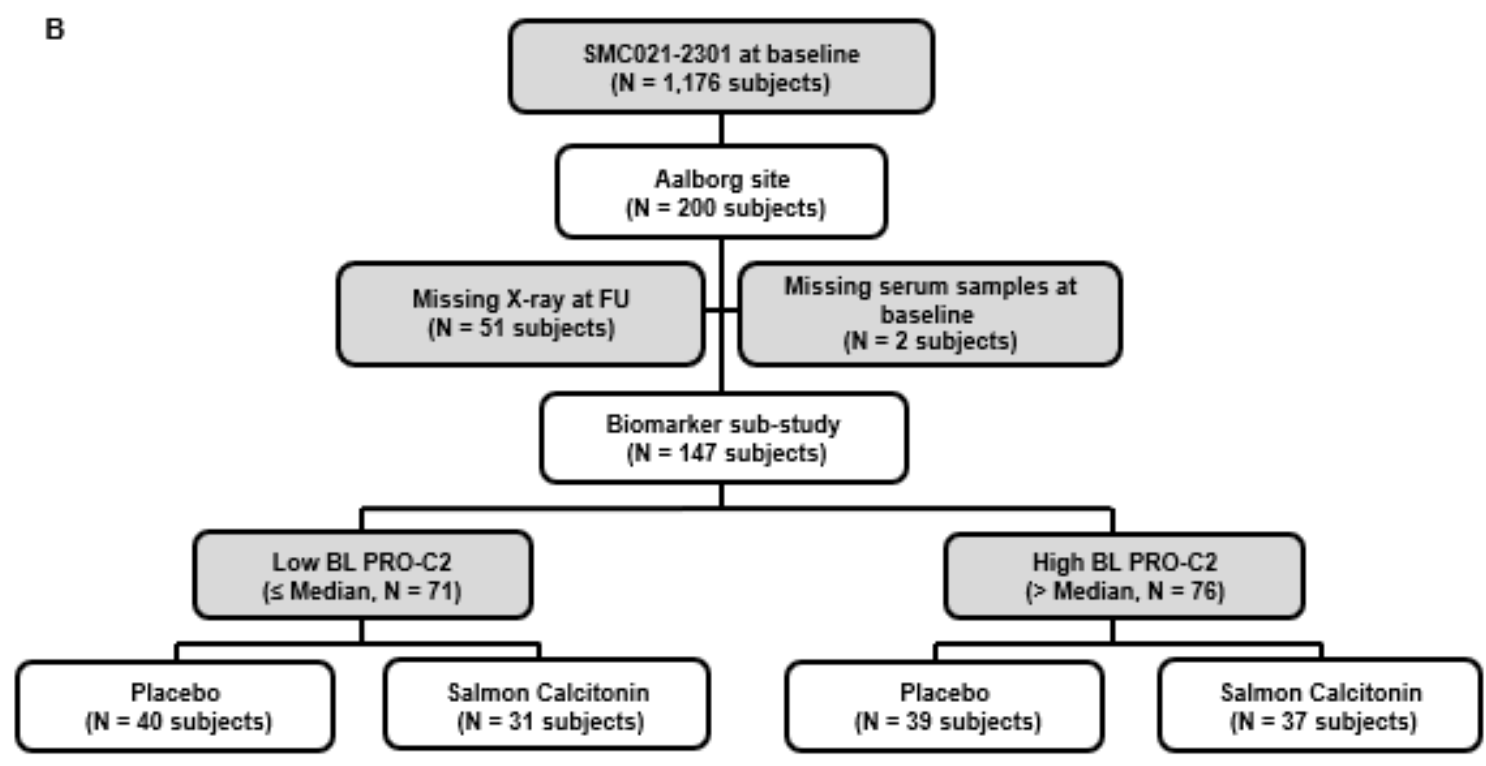

\section{Figure 1}

Flow diagrams for subjects included in the PRO-C2 post-hoc analyses. (A) The NYU cohort. (B) The SMC cohort.
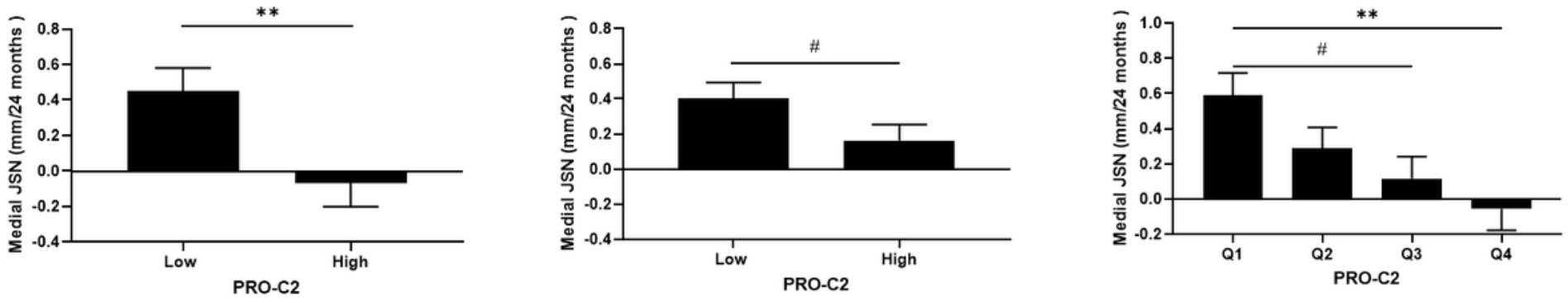

Figure 2 
Association between baseline levels of PRO-C2 and two-year radiographic progression (JSN). Patients were dichotomized, based on the median, to low and high levels of PRO-C2 and the degree of progression was compared in (A) the NYC and (B) the SMC cohort. (C) Assessment of "dose-response" of JSN as a function of PRO-C2 levels in the combined dataset. Analyses were adjusted for age, BMI, gender, VAS pain, and baseline medial JSW using two-way analysis of covariates (ANCOVA). Data are presented as estimated marginal means and standard error (SE). Asterisks: **, $p<0.01 ; \#, p<0.1$.

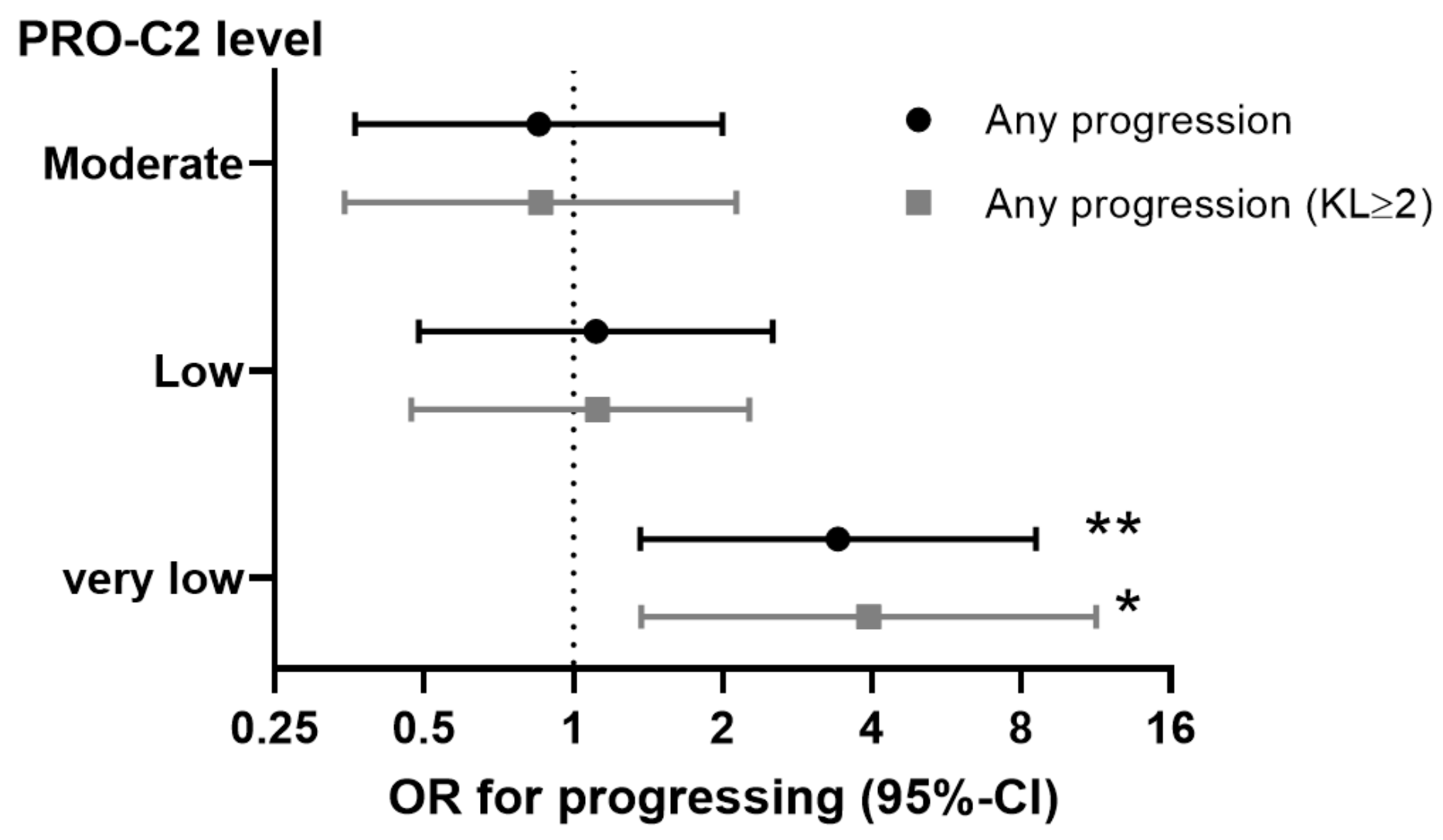

Figure 3

The odds ratio (OR) for progression in groups of patients with very low (Q1 from fig. 2), low (Q2) and moderate (Q3) compared to high (Q4) levels of baseline PRO-C2. Progression was defined as any progression (JSN $>0)$ over two-years in analyses including all patients $(n=185$, black) and patients with baseline $K L \geq 2$ ( $n=156$, Grey). Analyses were adjusted for age, BMI, gender, VAS pain, and baseline medial JSW using logistic regression. Data are presented as OR and 95\%-confidence intervals (CI). Asterisks: ${ }^{* *}, \mathrm{p}<0.01 ; *, \mathrm{p}<0.05$. 


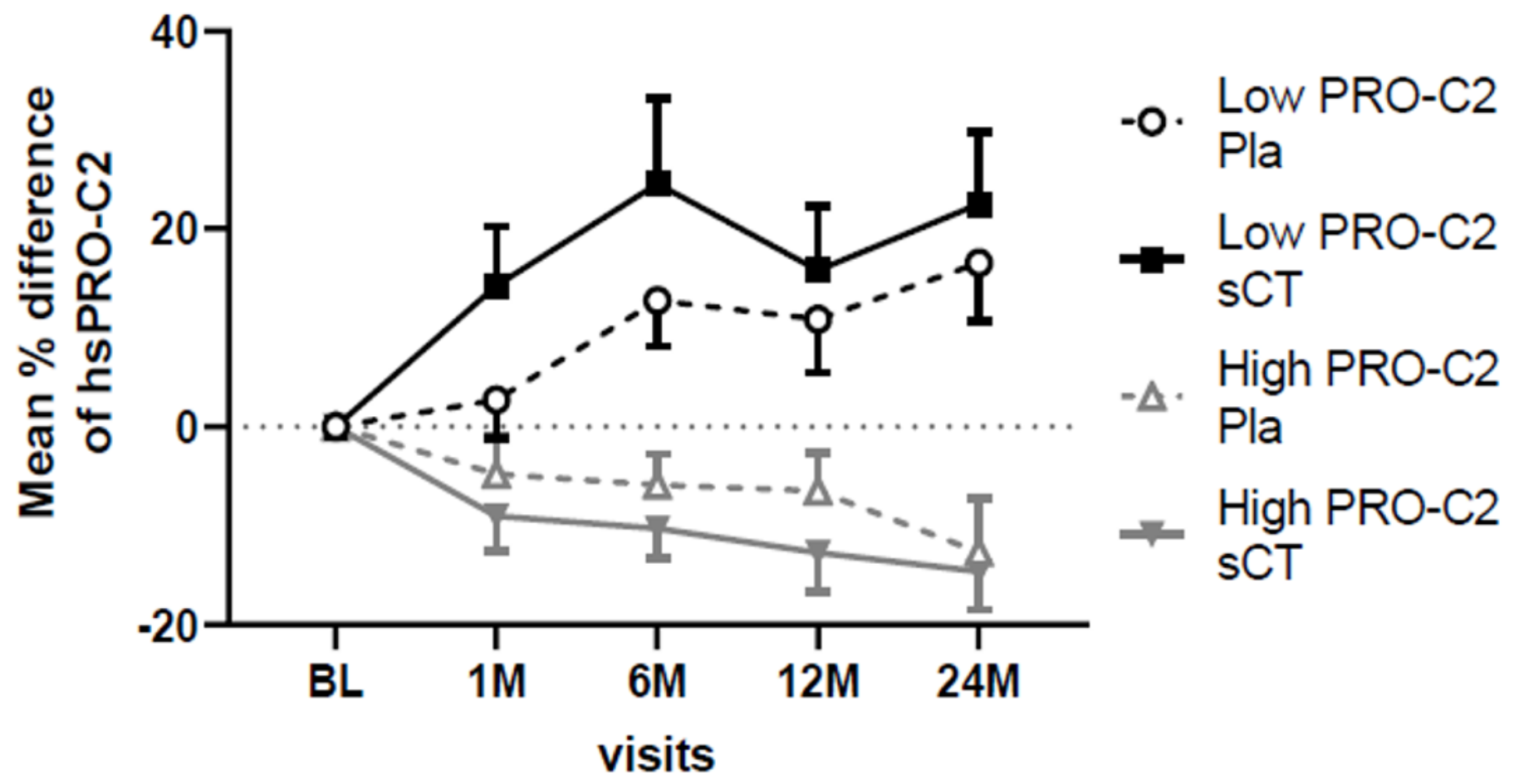

Figure 4

Pharmacodynamic effect of oral salmon calcitonin (sCT) vs. Placebo (Pla) on serum PRO-C2 levels over time in patients with low and high baseline PRO-C2. Data are shown as the mean (SEM) percentage difference from baseline. Time dependent effects were investigated by ANCOVA adjusting for BMI, sex, age, and baseline medial JSW.

Trauma-injury-driven phenotype $\longrightarrow$ High aggrecan degradation endotype (ARGS)
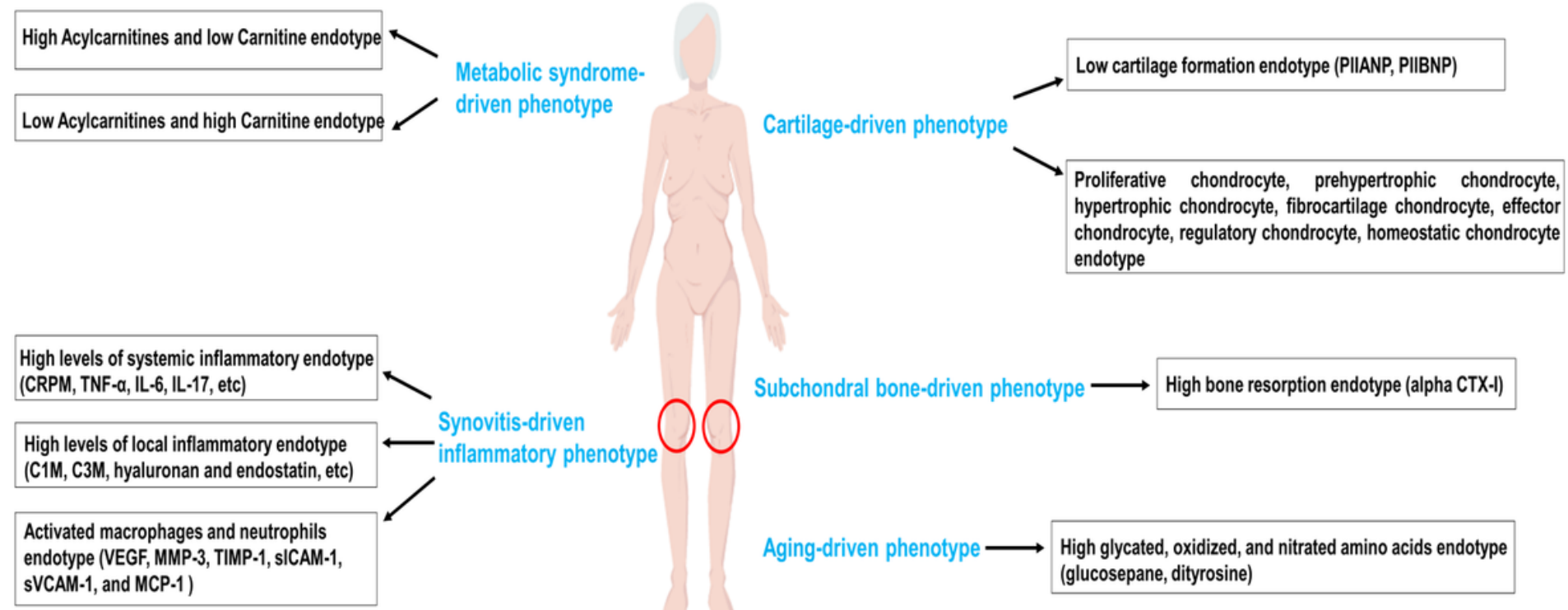


\section{Figure 5}

Emerging phenotypes and endotypes of osteoarthritis. ARGS: N-terminal neoepitope of the aggrecanasemediated aggrecan degradation fragment; C1M: MMP-derived collagen degradation neoepitopes of collagen I; C3M: MMP-derived collagen degradation neoepitopes of collagen III; CRPM: MMP-depended degradation product of C-reactive protein; CTX-I: C-terminal telopeptide of collagen I; IL-6: interleukin-6; IL17: interleukin-17; MCP-1: monocyte chemoattractant protein 1; MMP-3: matrix metalloproteinase-3; PIIBNP (N-terminal propeptide of procollagens IIB); sICAM-1: soluble intracellular adhesion molecule 1; sVCAM-1: soluble vascular cell adhesion molecule 1; TNF-a: tumour necrosis factor-alpha; TIMP-1: tissue inhibitor of metallopeptidase inhibitor 1; VEGF: vascular endothelial growth factor.

\section{Supplementary Files}

This is a list of supplementary files associated with this preprint. Click to download.

- SupplementaryS2.docx

- SupplementaryS3.docx

- SupplementaryS3.docx

- SupplementaryS4.docx

- SupplementaryS2.docx

- SupplementaryS4.docx

- SupplementaryS1.docx

- SupplementaryS1.docx 